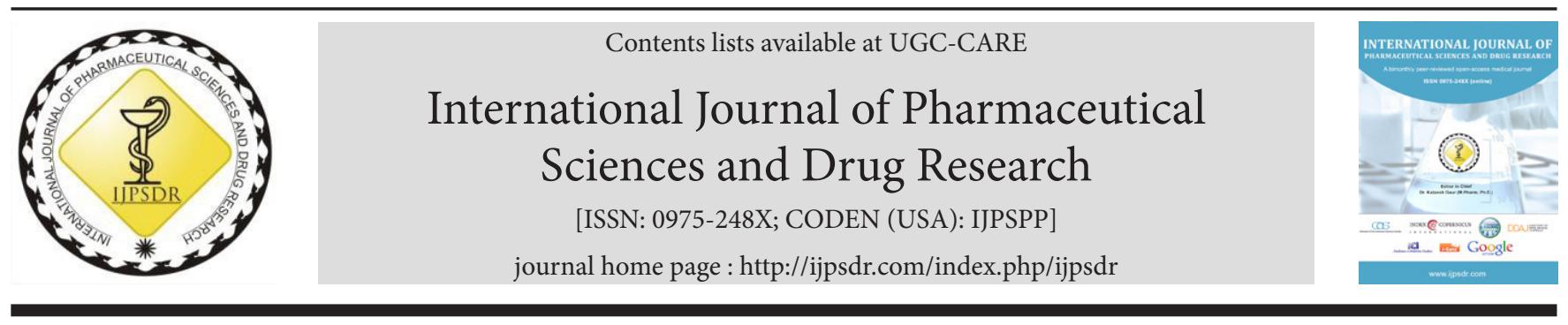

Research Article

\title{
Synthesis and Oral Hypoglycemic Activity of Some New Sulphonyl Linkage Thiazolidine-2, 4-diones
}

\author{
Laxman Kawale, Vandana Nade, Rohini Deshmukh, Mahesh Dumbare* \\ Department of Pharmaceutical Chemistry, MVPS's College of Pharmacy, Gangapur Road, Nashik-422002, Maharashtra, India.
}

\author{
ART I CLE INFO \\ Article history: \\ Received: 14 April, 2020 \\ Revised: 20 October, 2020 \\ Accepted: 28 October, 2020 \\ Published: 30 November, 2020 \\ Keywords: \\ Oral hypoglycemic activity, \\ Peroxisome proliferator-activated \\ receptor- $\gamma$, Streptozocin, \\ Thiazolidine-2,4-diones. \\ DOI: \\ 10.25004/IJPSDR.2020.120603
}

\begin{abstract}
A B S T R A C T
Thiazolidine-2, 4-diones are found to be better insulin-sensitizing agents via acting on peroxisome proliferator-activated receptor- $\gamma$ (PPAR- $\gamma$ ) and decrease blood glucose level in a diabetic patient. Therefore, in the present work, we synthesized 5-[4-(substituted) sulphonylbenzylidene] thiazolidine-2,4-diones and analyzed by proton, carbon-13 NMR, and Fourier-transform infrared (FTIR) spectroscopy. These newly synthesized thiazolidine-2,4-dione derivatives were evaluated for their oral hypoglycaemic activity on streptozocin-induced diabetes mellitus rats. All synthesized compounds were also further studied to determine their docking score, interactions with amino residues of PPAR- $\gamma$ (PDB ID: 2PRG protein), and predicated $\mathrm{ED}_{25}$ values. The oral hypoglycaemic activity results of all synthesized compounds showed a significant decrease in blood glucose level compared to a positive control group. The synthesized compounds were shown good hydrogen-bonding interactions with 2PRG protein, docking score, and predicted $\mathrm{ED}_{25}$ value compared with reference drug such as pioglitazone and rosiglitazone, respectively. Finally, the linkage of sulphonyl thiazolidine-2,4-diones may be used as a promising oral hypoglycemic agent.
\end{abstract}

\section{INTRODUCTION}

Type II diabetes (T2D) is a progressive disease characterized by insulin resistance in peripheral tissues or impaired insulin secretion by the pancreas, resulting in high blood glucose level, leading to developed several serious long-term microvascular and macrovascular complications decreases the quality life of diabetic patients. ${ }^{[1,2]}$ Therefore, it is essential to control the blood glucose level during the early stages of the disease. ${ }^{[3]}$ Therapy for type 2 diabetes mellitus (T2DM) has been aimed at improving glycemic control via a combination of diet, exercise and use of current therapeutic agents such as insulin, sulfonylureas, metformin, acarbose, thiazolidinediones (TZD's), dipeptidyl peptidase IV, glucagon-like peptide-1, glucokinase and so on. ${ }^{[4-6]}$ Among these current therapeutic agents, TZD's or glitazones are used as insulin-sensitizing agents via selective agonist's action on PPAR- $\gamma$, leads to an increase in insulin sensitivity and decrease high blood glucose level in the adipose, muscle and hepatic tissues. ${ }^{[7]}$

Now a day, a number of TZD's have been synthesized and clinically examined for their oral hypoglycemic activity. Unfortunately, ciglitazone, englitazone, darglitazone, KRP-297, CS-011 and CLX-0921 were discontinued in clinical trials. Mitoglitazone, troglitazone, and balaglitazone were processing in different clinical trials. Troglitazone and rosiglitazone were withdrawn from the market. Pioglitazone and lobeglitazone are still used therapeutically. ${ }^{[8]}$ Because of their additional beneficial effect confirmed during the treatment of a diabetic patient. These confirmed additional beneficial effects are improved the pancreatic $\beta$-cell function, improved endothelial function, lower blood pressure and decreased visceral fat, alanine aminotransferase, live fat, cardiovascular morbidity and mortality. ${ }^{[9,10]}$

\footnotetext{
*Corresponding Author: Dr. Mahesh Ramdas Dumbare

Address: Department of Pharmaceutical Chemistry, MVPS's College of Pharmacy, Gangapur Road, Nashik-422002, Maharashtra, India.

Email $\bowtie$ : ramvijay82dumbare@rediffmail.com

Tel.: +91-9270328703

Relevant conflicts of interest/financial disclosures: The authors declare that the research was conducted in the absence of any commercial or financial relationships that could be construed as a potential conflict of interest.

Copyright (C) 2020 Laxman Kawale etal. This is an open access article distributed under the terms of the Creative Commons Attribution- NonCommercialShareAlike 4.0 International License which allows others to remix, tweak, and build upon the work non-commercially, as long as the author is credited and the new creations are licensed under the identical terms.
} 
Despite their beneficial effects, the treatment of TZD's is also shown some adverse effects such as weight gain, edema, anemia, bone fractures, and cancer. ${ }^{[11]}$ Therefore, in the present work, we synthesized TZD's with sulphonyl linkage and evaluated for their oral hypoglycemic activity for the development of better and safer TZD's.

\section{Materials And Methods}

Reagents and various solvents purchased Fischer scientific and Modern industries. Thin layer chromatography (TLC) monitored all reactions carried out on $0.25 \mathrm{~mm}$ E-Merck silica gel plates (pre-coated aluminum sheets) with an appropriate solvent system used as mobile phase and UV cabinet (sometime also used iodine) used as visualizing agent. Melting points were determined in open capillary tubes or Elico melting point apparatus and were uncorrected. All final compounds were purified with the help of the recrystallization method. IR spectrums of all synthesized compounds were recorded on Perkin-Elmer AC-1 Spectrophotometer and Shimadzu FTIR-84005. ${ }^{1} \mathrm{HNMR}$ and ${ }^{13} \mathrm{CNMR}$ spectra were recorded in DMSO- $\mathrm{d}_{6}$ and $\mathrm{CDCL}_{3}$ solvent on Bruker advance II 400 NMR spectrometer. The chemical shifts values of proton and carbon-13 were measured with respect to TMS in $\delta \mathrm{ppm}$. Mass spectra were recorded on Water, Q-TOF micromass (ESI-MS).

\section{Synthetic Scheme and Synthesis Procedure}

The synthetic work was done with the help of a designed suitable synthetic route, mention in Scheme 1.

For the Synthesis of Thiazolidine-2,4-dione (1)

Thiazolidine-2,4-dione was synthesized as per the procedure reported in the literature. The product was purified by recrystallization using ethyl alcohol. ${ }^{[12]}$

For the Synthesis of 5-benzylidenethiazolidine-2,4-dione (2)

The synthesis of 5-benzylidenethiazolidine-2,4-dione was done using a Knoevenagel condensation reaction. The procedure of synthesis was reported in the literature. The product was purified by recrystallization using glacial acetic acid. ${ }^{[12]}$

For the Synthesis of 5-(4-chlorosulphonylbenzylidene) thiazolidine-2,4-dione (3)

The procedure of chlorosulphonation reaction was given in the literature. The product was filtered, dried and without purification, it was used for the next step. ${ }^{[12]}$

General Procedure for Synthesis of 5-[4-(substituted) sulphonylbenzylidene] thiazolidine-2,4-diones (4a-4h)

To an ice-cooled solution of substituted aniline (0.01 mol) in dichloromethane (20 mL) was added in a solution of dichloromethane $(10 \mathrm{~mL})$ containing 5-(4-chlorosulphonylbenzylidene) thiazolidine-2,4-dione $(0.01 \mathrm{~mol})$ and followed by addition of triethylamine $(0.014$ mol) in $250 \mathrm{ml}$ conical flask at $0^{\circ} \mathrm{C}$. The reaction mixture was stirred at $0^{\circ} \mathrm{C}$ for 15 minutes. The stirring was continued overnight at room temperature. The next day, the reaction mixture was extracted in dichloromethane and washed stepwise with $1 \mathrm{~N} \mathrm{HCl}, 5 \%$ sodium bicarbonate (2x), and brine solution. The extracted layer was dried over anhydrous magnesium sulfate and concentrate at room temperature to obtain crude compounds. All final crude compounds were purified in methanol. ${ }^{[13]}$

\section{Characterization and Spectral Interpretation}

\section{Thiazolidine-2,4-dione (1)}

Chemical formula: $\mathrm{C}_{3} \mathrm{H}_{3} \mathrm{NO}_{2} \mathrm{~S}$; Molecular mass: 117; Yield: 85\%; Rf value: 0.60 ; M.P: $123-125^{\circ} \mathrm{C}$; IR( $\left(\mathrm{KBr}_{\mathrm{Cm}}{ }^{-1}\right): 3123(\mathrm{~N}-$ HStr., amide), 3036,2947,2817(C-HStr.,thiazolidine-2,4dione), 1763,1647( $\mathrm{C}=0$ str., thiazolidine-2,4-dione ), 1338,1157(C-NStr., thiazolidine-2,4-dione); ${ }^{1} \mathrm{H} \mathrm{NMR}\left(\mathrm{CDCl}_{3}\right.$, $\delta \mathrm{ppm})$ : 8.61(s, $1 \mathrm{H}, \mathrm{NH}), 4.05\left(\mathrm{~s}, 2 \mathrm{H},-\mathrm{CH}_{2}-\right)$; $\mathrm{MS}(\mathrm{m} / \mathrm{z})$ : 117.01.

\section{5-Benzylidenethiazolidine-2,4-dione (2)}

Chemical formula: $\mathrm{C}_{10} \mathrm{H}_{7} \mathrm{NO}_{2} \mathrm{~S}$; Molecular mass: 205; Yield: 91\%; Rf value: 0.40; M.P: $240-242^{\circ} \mathrm{C}$; IR $\left(\mathrm{KBr}, \mathrm{cm}^{-1}\right)$ : $3150(\mathrm{~N}-$ HStr., amide), 3050(C-HStr., Ar), 2800 (C-HStr.,thiazolidine2,4-dione), 1770,1693(C=0Str.,thiazolidine-2,4-dione), 1600,1470 (C=CStr.,Ar), 1358,1137

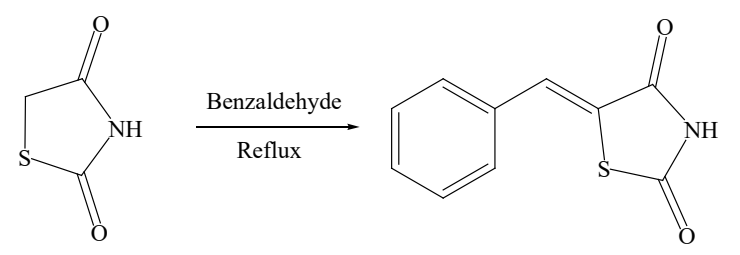

Thiazolidine-2,4-dione (1) 5-benzylidenethiazolidine-2,4-dione (2) $\begin{gathered}\text { Stirring } 0-5^{\circ} \mathrm{C} \text { and } \\ \text { then reflux } 1 \mathrm{~h}\end{gathered} \mid$ Chlorosulphonic acid<smiles>O=C1NC(=O)/C(=C/c2ccc(S(=O)(=O)Cl)cc2)S1</smiles>

5-(4-chlorosulphonylbenzylidene)thiazolidine-2,4-dione (3) $\begin{gathered}\text { Stirring, dichlromethane, } \\ \text { triethylamine, overnight }\end{gathered} \mid$ Substituted aniline

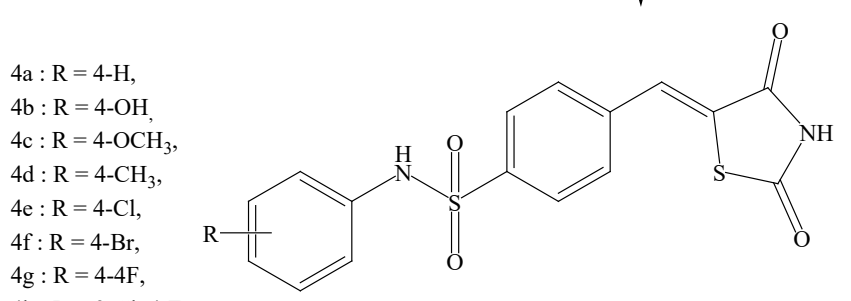

4h: $\mathrm{R}=3-\mathrm{Cl}, 4-\mathrm{F} \quad$ Fig. 1: Synthesis of target compounds (4a-4h)

Scheme 1: Synthetic scheme of synthesis of sulphonyl linkage thiazolidine-2,4-diones. 
Hypoglycemic Activity of Newly Synthesis Sulphonyl Thiazolidine-2,4-diones.

(C-NStr.,thiazolidine-2,4-dione); ${ }^{1} \mathrm{HNMR}\left(\mathrm{DMSOd}_{6}, \delta \mathrm{ppm}\right)$ : 12.57(s, $1 \mathrm{H}, \mathrm{NH}), 7.79(\mathrm{~s}, 1 \mathrm{H}$, benzylidene), $7.60(\mathrm{~d}, 2 \mathrm{H}$, Ar-CH), 7.50(d, 2H, Ar-CH), 7.49(t, 1H, Ar-CH); MS (m/z): 205.04 .

\section{5-(4-Chlorosulphonylbenzylidene)thiazolidine-2,4-dione}

(3)

Chemical formula: $\mathrm{C}_{10} \mathrm{H}_{6} \mathrm{ClNO}_{4} \mathrm{~S}_{2}$; Molecular mass: 303; Yield: $68 \%$; Rf value: 0.30; M.P: $180-181^{\circ} \mathrm{C}$; $\mathrm{IR}\left(\mathrm{KBr}, \mathrm{cm}^{-1}\right)$ : 3217(N-HStr., amide), 3025(C-HStr., Ar), 2800 (C-HStr., thiazolidine-2,4-dione), 1743,1674(C=0Str., thiazolidine2,4-dione), 1604(C=CStr.,Ar),1373 (S=0 Asymmetric str., sulphonyl), 1290 (C-NStr., thiazolidine-2,4-dione), 1165(S=0 Symmetric str., sulphonyl); ${ }^{1} \mathrm{HNMR}\left(\operatorname{DMSOd}_{6}\right.$, Sppm): 12.64(s, 1H, NH), 7.77(s, 1H, benzylidene), 7.72(d, 2H, Ar-CH), 7.57 (d, 2H, Ar-CH). MS (m/z): 303.74.

5-[4-(Aminophenyl)sulphonylbenzylidene] thiazolidine-2,4dione (4a)

Chemical formula: $\mathrm{C}_{16} \mathrm{H}_{12} \mathrm{~N}_{2} \mathrm{O}_{4} \mathrm{~S}_{2}$; Molecular mass:360; Yield: $20 \%$; Rf value: 0.62; M.P: $230-232^{\circ} \mathrm{C}$; IR $\left(\mathrm{KBr}, \mathrm{cm}^{-1}\right)$ : 3429 (N-HStr., thiazolidine-2,4-dione), 3245(N-HStr., sulphonamide), 2985(C-HStr., Ar) 1743,1693(C=OStr., thiazolidine-2,4-dione), 1603,1492 (C=CStr., Ar), 1342(S=0 Asymmetric str., Sulphonyl), 1242(C-NStr., thiazolidine2,4-dione), 1126(S=0 Symmetric str., Sulphonyl), 1180(CNStr., amine); $\left.{ }^{1} \mathrm{H} \mathrm{NMR} \mathrm{(DMSOd}_{6}, \delta \mathrm{ppm}\right): 9.25(\mathrm{~s}, 1 \mathrm{H}, \mathrm{N}-\mathrm{H})$, $8.20(\mathrm{~s}, 1 \mathrm{H}, \mathrm{N}-\mathrm{H}), 7.84(\mathrm{~d}, 2 \mathrm{H}, \mathrm{Ar}), 7.76(\mathrm{~d}, 2 \mathrm{H}, \mathrm{Ar}), 7.73(\mathrm{~d}, 2 \mathrm{H}$, $\mathrm{Ar}), 7.64(\mathrm{~s}, 1 \mathrm{H}$, benzylidene), 7.08(d, $2 \mathrm{H}, \mathrm{Ar}), 7.0(\mathrm{t}, 1 \mathrm{H}, \mathrm{Ar})$;

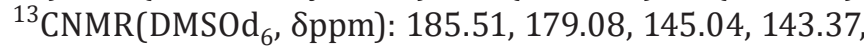
$143.25,142.80,134.66,134.40,134.00,132.36,129.37$, 126.63, 125.53, 119.09; MS(m/z): 360.29.

\section{5-[4-(4-Hydroxyaminophenyl)sulphonylbenzylidene] thiazolidine-2,4-dione (4b)}

Chemical formula: $\mathrm{C}_{16} \mathrm{H}_{12} \mathrm{~N}_{2} \mathrm{O}_{5} \mathrm{~S}_{2}$; Molecular mass:376; Yield: 72\%; Rf value: 0.60; M.P: $215-216^{\circ} \mathrm{C}$; IR $\left(\mathrm{KBr}, \mathrm{cm}^{-}\right.$ ${ }^{1}$ ): 3568(0-HStr., aminophenol), 3409 (N-HStr., thiazolidine-2,4-dione), 3240 (N-H Str.,sulphonamide), 3008,2923(C-HStr.,Ar) 1750,1693(C=OStr., thiazolidine2,4-dione), 1600,1475(C=CStr., Ar), 1327(S=0 Asymmetric str., sulphonyl), 1226(C-NStr., thiazolidine-2,4-dione), 1168 (S=0 Symmetric str., sulphonyl), 1010 (C-NStr., amine); ${ }^{1} \mathrm{H}$ NMR(DMSOd $\left.{ }_{6}, \delta p p m\right): 9.40(\mathrm{~s}, 1 \mathrm{H}, \mathrm{N}-\mathrm{H}), 8.10(\mathrm{~s}, 1 \mathrm{H}, \mathrm{N}-\mathrm{H})$, 7.84(d, 2H, Ar), 7.62(d, 2H, Ar), 7.54(s, 1H, benzylidene), 6.40(d, 2H, Ar), 6.25(d, 2H, Ar), 4.5(bs, 1H, OH).

\section{5-[4-(4-Methoxyaminophenyl)sulphonylbenzylidene] thiazolidine-2,4-dione (4c)}

Chemical formula: $\mathrm{C}_{17} \mathrm{H}_{14} \mathrm{~N}_{2} \mathrm{O}_{5} \mathrm{~S}_{2}$; Molecular mass:390; Yield: 54\%; Rf value: 0.54; M.P: $200-202^{\circ} \mathrm{C}$; IR $\left(\mathrm{KBr}, \mathrm{cm}^{-1}\right)$ : 3434(N-HStr., thiazolidine-2,4-dione), 3204(N-HStr., sulphonamide), 2989(C-HStr.,Ar), 2773(C-HStr., $\mathrm{CH}_{3}$ ) 1747,1700 (C=0 Str., thiazolidine-2,4-dione), 1638, 1492(C=CStr., Ar), 1330( $\mathrm{S}=0$ Asymmetric str., sulphonyl), 1230(C-NStr., thiazolidine-2,4-dione), 1135(S=0 Symmetric str., sulphonyl), 1006(C-NStr., amine); ${ }^{1} \operatorname{HNMR(D{}^{2}}{ } d_{6}$,
Sppm): $9.30(\mathrm{~s}, 1 \mathrm{H}, \mathrm{N}-\mathrm{H}), 8.40(\mathrm{~s}, 1 \mathrm{H}, \mathrm{N}-\mathrm{H}), 7.70(\mathrm{~d}, 2 \mathrm{H}, \mathrm{Ar})$, $7.49(\mathrm{~d}, 2 \mathrm{H}, \mathrm{Ar}), 7.27(\mathrm{~s}, 1 \mathrm{H}$, benzylidene), 7.27(d, 2H, Ar), $7.00(\mathrm{~d}, 2 \mathrm{H}, \mathrm{Ar}), 3.70\left(\mathrm{~s}, 1 \mathrm{H}, \mathrm{OCH}_{3}\right)$. MS(m/z): 389.19 .

\section{5-[4-(4-Methylaminophenyl)sulphonylbenzylidene] thiazolidine-2,4-dione (4d)}

Chemical formula: $\mathrm{C}_{17} \mathrm{H}_{14} \mathrm{~N}_{2} \mathrm{O}_{4} \mathrm{~S}_{2}$; Molecular mass:374; Yield: 69\%; Rf value: 0.73; M.P: $244-246^{\circ} \mathrm{C}$; IR( $\left.\mathrm{KBr}, \mathrm{cm}^{-1}\right)$ : 3463(N-HStr., thiazolidine-2,4-dione), 3255(N-HStr., sulphonamide), 3051(C-HStr., Ar), 2850 (C-HStr., $\mathrm{CH}_{3}$ ), 1739,1712 (C=OStr., thiazolidine-2,4-dione), 1604, 1492(C=CStr.,Ar), 1338(S=0 Asymmetric str., sulphonyl), 1288(C-NStr., thiazolidine-2,4-dione), 1157(S=0 Symmetric str., sulphonyl), 1091(C-NStr., amine); ${ }^{1} \mathrm{HNMR}\left(\mathrm{DMSOd}_{6}\right.$, Sppm): $9.10(\mathrm{~s}, 1 \mathrm{H}, \mathrm{N}-\mathrm{H}), 8.20(\mathrm{~s}, 1 \mathrm{H}, \mathrm{N}-\mathrm{H}), 7.62(\mathrm{~d}, 2 \mathrm{H}, \mathrm{Ar})$, 7.48(d, 2H, Ar), 7.25(s, 1H, benzylidene), 6.80(d, 2H, Ar), $6.60(\mathrm{~d}, 2 \mathrm{H}, \mathrm{Ar}), 2.30\left(\mathrm{~s}, 1 \mathrm{H}, \mathrm{CH}_{3}\right)$.

\section{5-[4-(4-Chloroaminophenyl)sulphonylbenzylidene] thiazolidine-2,4-dione (4e)}

Chemical formula: $\mathrm{C}_{16} \mathrm{H}_{11} \mathrm{ClN}_{2} \mathrm{O}_{4} \mathrm{~S}_{2}$; Molecular mass:393; Yield:69\%; Rf value:0.62; M.P: $195-197^{\circ} \mathrm{C}$; IR $\left(\mathrm{KBr}, \mathrm{cm}^{-1}\right)$ : 3425(N-HStr., thiazolidine-2,4-dione), 3235(N-HStr., sulphon- amide), 2985(C-HStr., Ar), 1743,1703(C=0 Str., thiazolidine-2,4-dione), 1608,1492 (C=C Str., Ar), 1342(S=0 Asymmetric str., sulphonyl), 1280(C-NStr., thiazolidine2,4-dione), 1180(S=0 Symmetric str., sulphonyl), 1085(CNStr., amine). 771(C-ClStr.); ${ }^{1} \mathrm{HNMR}$ (DMSOd $\left.{ }_{6}, \delta \mathrm{ppm}\right)$ : 9.50(s, $1 \mathrm{H}, \mathrm{N}-\mathrm{H}), 8.53(\mathrm{~s}, 1 \mathrm{H}, \mathrm{N}-\mathrm{H}), 7.85(\mathrm{~d}, 2 \mathrm{H}, \mathrm{Ar}), 7.50(\mathrm{~d}, 2 \mathrm{H}$, Ar), 7.49(s, 1H, benzylidene), 7.30(d, 2H, Ar), 7.09(d, 2H, Ar).

\section{5-[4-(4-Bromoaminophenyl)sulphonylbenzylidene] thiazolidine-2,4-dione (4f)}

Chemical formula: $\mathrm{C}_{16} \mathrm{H}_{11} \mathrm{BrN}_{2} \mathrm{O}_{4} \mathrm{~S}_{2}$; Molecular mass:437; Yield:62\%; Rf value:0.41; M.P: $149-152^{\circ} \mathrm{C}$; IR( $\left.\mathrm{KBr}, \mathrm{cm}^{-1}\right)$ : 3387 (N-HStr., thiazolidine-2,4-dione), 3232(N-HStr., sulphon- amide), 3055(C-HStr., Ar) 1743,1681(C=0Str., thiazolidine-2,4-dione), 1612,1411(C=C Str., Ar), 1327(S=0 Asymmetric str., sulphonyl), 1219(C-N Str., thiazolidine2,4-dione), 1141( $\mathrm{S}=0$ Symmetric str., sulphonyl), 1033(CNStr., amine), 686(C-BrStr.); ${ }^{1} \mathrm{HNMR}\left(\mathrm{DMSOd}_{6}, \delta \mathrm{ppm}\right)$ : 9.61(s, $1 \mathrm{H}, \mathrm{N}-\mathrm{H}), 8.57(\mathrm{~s}, 1 \mathrm{H}, \mathrm{N}-\mathrm{H}), 7.84(\mathrm{~d}, 2 \mathrm{H}, \mathrm{Ar}), 7.62(\mathrm{~d}$, $2 \mathrm{H}, \mathrm{Ar}), 7.59(\mathrm{~s}, 1 \mathrm{H}$, benzylidene), 7.33(d, 2H, Ar), 7.11(d, $2 \mathrm{H}, \mathrm{Ar}) ;{ }^{13} \mathrm{CNMR}\left(\mathrm{DMSOd}_{6}\right.$, Sppm): 172.11, 170.18, 147.00, $139.22,137.96,136.58,131.58,131.08,131.01,130.56$, $129.63,127.09,126.72,121.86,116.56,115.82,107.15$; MS (m/z): 439.03.

\section{5-[4-(4-Fluroaminophenyl)sulphonylbenzylidene] thiazolidine-2,4-dione (4g)}

Chemical formula: $\mathrm{C}_{16} \mathrm{H}_{11} \mathrm{FN}_{2} \mathrm{O}_{4} \mathrm{~S}_{2}$; Molecular mass:378; Yield:72\%; Rf value:0.52; M.P: $175-178^{\circ} \mathrm{C}$; $\operatorname{IR}\left(\mathrm{KBr}, \mathrm{cm}^{-1}\right)$ : 3332 (N-HStr., thiazolidine-2,4-dione), 3240 (N-HStr., sulphonamide), 2931(C-HStr., Ar), 1735,1681(C=0Str., thiazolidine-2,4-dione), 1612,1404(C=CStr., Ar), 1319(S=0 Asymmetric str., sulphonyl), 1288(C-NStr., thiazolidine2,4-dione), 1188(S=0 Symmetric str., sulphonyl), 1126(C- 
NStr., amine), 1033(C-FStr.); ${ }^{1} \mathrm{HNMR}$ DMSOd $_{6}, \delta$ ppm): $10.21(\mathrm{~s}, 1 \mathrm{H}, \mathrm{N}-\mathrm{H}), 8.90(\mathrm{~s}, 1 \mathrm{H}, \mathrm{N}-\mathrm{H}), 7.86(\mathrm{~d}, 2 \mathrm{H}, \mathrm{Ar}), 7.65(\mathrm{~d}, 2 \mathrm{H}$, Ar), 7.59(s, 1H, benzylidene), 7.33(d, 2H, Ar), 7.14(d, 2H, Ar).

\section{5-[4-(3-Chloro, 4-fluroaminophenyl)sulphonylbenzylidene] thiazolidine-2,4-dione (4h)}

Chemical formula: $\mathrm{C}_{16} \mathrm{H}_{10} \mathrm{ClFN}_{2} \mathrm{O}_{4} \mathrm{~S}_{2}$; Molecular mass:411; Yield:66\%; Rf value:0.54; M.P: $215-217^{\circ} \mathrm{C}$; $\mathrm{IR}\left(\mathrm{KBr}, \mathrm{cm}^{-1}\right)$ : 3371(N-HStr., thiazolidine-2,4-dione), 3232(N-HStr., sulphonamide), 3055,2939(C-HStr., Ar), 1735,1681(C=OStr., thiazolidine-2,4-dione), 1612, 1404(C=CStr., Ar), 1319(S=0 Asymmetric str., sulphonyl), 1219(C-NStr., thiazolidine-2,4dione), 1126(S=0 Symmetric str., sulphonyl), 1126(C-NStr., amine), 1033(C-FStr.), 771(C-ClStr.); ${ }^{1} \mathrm{HNMR}$ DMSOd $_{6}$, Sppm): 10.62(s, $1 \mathrm{H}, \mathrm{N}-\mathrm{H}), 9.25(\mathrm{~s}, 1 \mathrm{H}, \mathrm{N}-\mathrm{H}), 7.86(\mathrm{~d}, 2 \mathrm{H}, \mathrm{Ar})$, 7.76(d, 2H, Ar), 7.57(s, 1H, benzylidene), 7.50(s, 1H, Ar), 7.46(d, 2H, Ar), 7.31(d, 2H, Ar); MS(m/z): 411.10.

\section{Streptozotocin-induced Diabetes Mellitus (DM)}

All synthesized compounds were evaluated on streptozotocin-induced diabetes mellitus. Male Wistar rats (150-210 g) were housed in polycarbonate cages in an animal room with $12 \mathrm{~h}$ day-night cycle at a temperature of $22 \pm 2^{\circ} \mathrm{C}$ and humidity of $45-60 \%$. All animals were fed with pelleted rats chow and water during the experiment. The required animals for the present study were divided into four groups such as normal control group (NCG), positive control group (PCG), standard control group (SCG), and test control group (TCG). Each group (i.e., NCG, PCG, and SCG) and sub-groups of TCG (TCG4a-4h) were included six rats per group. Diabetes conditions were induced in Male albino rats of Wistar strain of all groups (except NCG) by the administration of intraperitoneal injection of freshly prepared streptozotocin $(60 \mathrm{mg} / \mathrm{kg}$ body weight) solution in $0.1 \mathrm{M}$ citrate buffer ( $\mathrm{pH} 4.5$ ) of cold solution to the overnight fasted rats. Whereas, NCG of rats was injected with only $0.1 \mathrm{M}$ citrate buffer $(\mathrm{pH}$ $4.5)$ solutions. On the $3^{\text {rd }}$ of streptozocin treatment, an increase in blood glucose levels above $250 \mathrm{mg} / \mathrm{dL}$. These rats were considered as diabetic rats. The treatment of test compounds was started on the fourth day to its respective TCG by oral route at desired dose levels, $100 \mathrm{mg} / \mathrm{kg}$ body weight, and considered as the first day of treatment. Simultaneously, a standard drug containing pioglitazone was also administered to its respective SCG by oral route at the desired dose level, $20 \mathrm{mg} / \mathrm{kg} /$ body weight and monitoring the blood glucose levels of all groups at different time interval such as 2 and 5 hours. ${ }^{[14]}$

\section{Molecular Docking Studies}

Docking studies on LigPrep treated ligands were carried out to predict the binding pocket of 2PRG using the docking program, and glide used a series of hierarchical filters to search for possible locations for the ligand in the active site region of the receptor. For the grid-based ligand docking, the receptor grid generation file was used. For protein structure, during the calculations of active site was determined within a grid box of $X=49.19, Y=-37.08, Z=$ 19.00 and grid box range $X=24.30, Y=24.30, Z=24.30$ with a (default inner box) grid box ligand range $X=10, Y=10$, $\mathrm{Z}=10$ was centered on the corresponding co-crystallized ligand. Default parameters were used, and no constraints were included. All prepared ligands were docked using standard precision (SP). The docking analyses of synthetic compounds, pioglitazone, and rosiglitazone were carried out on docking software of Schrodinger Maestro Module Glide Version 9.2. ${ }^{[15]}$

\section{Molecular 3D-QSAR Studies}

\section{Selection of Reference Compounds}

A data set of 33 reference compounds of 5-substituted thiazolidine-2,4-diones series were selected for the comparative molecular field analysis (CoMFA) model. All reference compounds were selected from the literature, ${ }^{[16]}$ based on their most significant oral hypoglycemic activities were tested on genetically obese and diabetic yellow $\mathrm{KK}$ mice and expressed in $\mathrm{pED}_{25}$, which is -log of the effective molar dose required to reduce blood glucose by $25 \%$. The dataset of all 33 reference compounds were sorted randomly into 26 compounds of training set molecules (TSM), and 7 compounds of test set molecules (TSM) in the process of model refinement for CoMFA reported herein Tables 1-3.

\section{Structure Preparation and Alignment}

3D structures of all reference compounds and all synthesized compounds were constructed using Sketch Molecule module. Further, the selection of active compound, number 33 as a template molecule from reference compounds. All reference compounds were built by using the coordinates of the ligand present in the crystal structure of PPAR- $\gamma$ receptor (PDB ID: 2PRG). These reference compounds were later minimized by applying thetripos molecular mechanics force field with a conjugate gradient method. The minimization was terminated when the energy gradient convergence criterion $0.05 \mathrm{kcal} / \mathrm{mol}$ was reached or when 10,000 steps minimization cycle was exceeded. The fragment was used as the common structure in Gasteiger-Huckel charges were applied to all reference compounds (Fig. 1a).

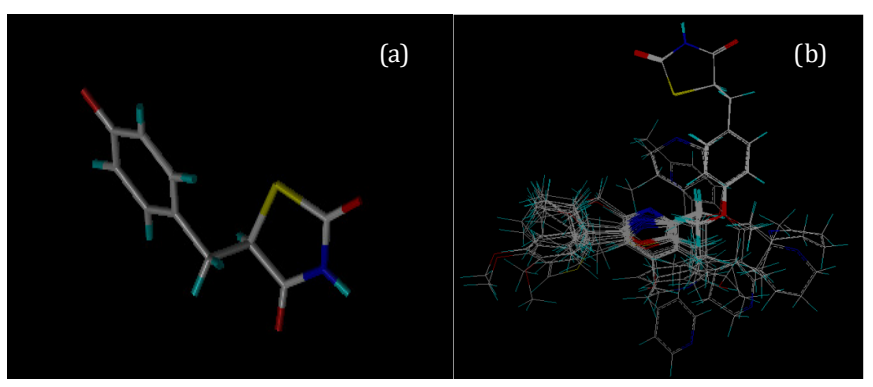

Fig. 1: a) Common structure for alignment b) Alignment of all reference compounds calculation of CoMFA descriptors 
Table 1: Actual and predicted activity of selected 33 reference compounds calculated by CoMFA.

\begin{tabular}{|c|c|c|c|c|c|c|c|c|}
\hline Sr. No. & $n$ & $A$ & $B$ & $C$ & $D$ & $E$ & $p E D_{25}{ }^{\#}$ & $p E D_{25}{ }^{* *}$ \\
\hline TSM1 & 2 & $\mathrm{~N}$ & $\mathrm{CH}$ & $\mathrm{CH}$ & $\mathrm{CH}$ & $\mathrm{CH}$ & 1.830 & 1.883 \\
\hline TSM 2 & 2 & $\mathrm{~N}$ & $\mathrm{C}-\mathrm{CH}_{3}$ & $\mathrm{CH}$ & $\mathrm{CH}$ & $\mathrm{CH}$ & 1.930 & 1.903 \\
\hline TSM 3 & 1 & $\mathrm{CH}$ & $\mathrm{N}$ & $\mathrm{CH}$ & $\mathrm{CH}$ & $\mathrm{CH}$ & 0.800 & 0.724 \\
\hline TSM $^{*}$ & 2 & $\mathrm{CH}$ & $\mathrm{N}$ & $\mathrm{CH}$ & $\mathrm{CH}$ & $\mathrm{CH}$ & 1.520 & 0.950 \\
\hline TSM5 $^{*}$ & 3 & $\mathrm{CH}$ & $\mathrm{N}$ & $\mathrm{CH}$ & $\mathrm{CH}$ & $\mathrm{CH}$ & 0.840 & 1.300 \\
\hline TSM6 & 2 & $\mathrm{CH}$ & $\mathrm{CH}$ & $\mathrm{N}$ & $\mathrm{CH}$ & $\mathrm{CH}$ & 0.820 & 0.760 \\
\hline TSM7 & 2 & $\mathrm{CH}$ & $\mathrm{CH}$ & $\mathrm{N}$ & $\mathrm{CH}$ & $\mathrm{C}-\mathrm{CH}_{3}$ & 0.840 & 0.975 \\
\hline TSM8 & 1 & $\mathrm{~N}$ & $\mathrm{C}-\mathrm{CH}_{3}$ & $\mathrm{CH}$ & $\mathrm{CH}$ & $\mathrm{CH}$ & 1.040 & 0.974 \\
\hline TSM9* & 2 & $\mathrm{CH}$ & $\mathrm{CH}$ & $\mathrm{CH}$ & $\mathrm{CH}$ & $\mathrm{CH}$ & 1.040 & 1.210 \\
\hline TSM10 & 2 & $\mathrm{~N}$ & $\mathrm{CH}$ & $\mathrm{CH}$ & $\mathrm{CH}$ & $\mathrm{C}-\mathrm{CH}_{3}$ & 1.230 & 1.357 \\
\hline TSM11 & 2 & $\mathrm{~N}$ & $\mathrm{CH}$ & $\mathrm{CH}$ & $\mathrm{C}-\mathrm{CH}_{3}$ & $\mathrm{CH}$ & 1.230 & 1.183 \\
\hline TSM12 & 2 & $\mathrm{~N}$ & $\mathrm{CH}$ & $\mathrm{C}-\mathrm{CH}_{3}$ & $\mathrm{CH}$ & $\mathrm{CH}$ & 1.230 & 1.407 \\
\hline TSM13 & 2 & $\mathrm{~N}$ & $\mathrm{CH}$ & $\mathrm{C}-\mathrm{CH}_{2}-\mathrm{CH}_{3}$ & $\mathrm{CH}$ & $\mathrm{CH}$ & 1.770 & 1.609 \\
\hline TSM14* & 2 & $\mathrm{~N}$ & $\mathrm{C}-\mathrm{CH}_{3}$ & $\mathrm{CH}$ & $\mathrm{C}-\mathrm{CH}_{3}$ & $\mathrm{CH}$ & 1.930 & 1.370 \\
\hline TSM15 & 2 & $\mathrm{~N}$ & $\mathrm{C}-\mathrm{CH}_{2}-\mathrm{OH}$ & $\mathrm{CH}$ & $\mathrm{CH}$ & $\mathrm{CH}$ & 0.860 & 0.931 \\
\hline TSM16 & 2 & $\mathrm{~N}$ & $\mathrm{C}-\mathrm{CH}_{3}$ & $\mathrm{CH}$ & $\mathrm{C}-\mathrm{OH}$ & $\mathrm{CH}$ & 0.860 & 0.863 \\
\hline
\end{tabular}

${ }^{*}$ Test set molecules and remaining are training set molecules, ${ }^{*}$ Actual activity, ${ }^{* *}$ Predicated activity.

Table 2: Actual and predicted activity of selected 33 reference compounds calculated by CoMFA.

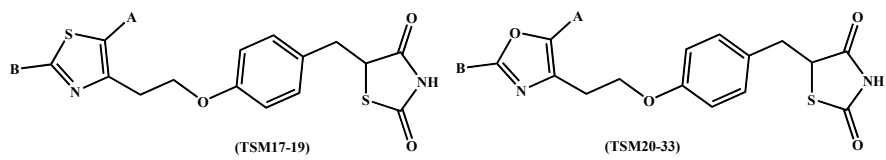

\begin{tabular}{|c|c|c|c|c|}
\hline Sr. No. & $A$ & $B$ & $p E D_{25}{ }^{\#}$ & $p E D_{25}{ }^{* *}$ \\
\hline TSM17 & $\mathrm{H}$ & $\mathrm{CH}_{3}$ & 1.940 & 1.853 \\
\hline TSM18 & $\mathrm{H}$ & -Cyclohexyl & 2.720 & 2.554 \\
\hline TSM19 & $\mathrm{H}$ & -Phenyl & 2.910 & 2.786 \\
\hline TSM20 & $\mathrm{H}$ & $-\mathrm{CH}_{3}$ & 1.840 & 2.003 \\
\hline TSM21 & $\mathrm{H}$ & $-\mathrm{CH}_{2}-\mathrm{CH}_{2}-\mathrm{CH}_{3}$ & 2.590 & 2.561 \\
\hline TSM22 & $-\mathrm{CH}_{3}$ & $-\mathrm{CH}_{3}$ & 2.250 & 2.227 \\
\hline TSM23 & $-\mathrm{CH}_{2}-\mathrm{CH}_{3}$ & $-\mathrm{CH}_{3}$ & 2.150 & 2.134 \\
\hline TSM24 & $\mathrm{H}$ & -Cyclohexyl & 2.870 & 2.861 \\
\hline TSM25 & $\mathrm{H}$ & -Phenyl & 3.100 & 3.039 \\
\hline TSM26 & $-\mathrm{CH}_{3}$ & -Phenyl & 3.620 & 3.553 \\
\hline TSM27* & $-\mathrm{CH}_{2}-\mathrm{CH}_{3}$ & -Phenyl & 3.420 & 3.440 \\
\hline TSM28 & $-\mathrm{CH}_{3}$ & -Cyclohexyl & 3.290 & 3.479 \\
\hline TSM29* & $-\mathrm{CH}_{3}$ & -Furyl & 2.990 & 2.960 \\
\hline TSM30* & $-\mathrm{CH}_{3}$ & -Thienyl & 3.370 & 3.310 \\
\hline TSM31 & $-\mathrm{CH}_{3}$ & - 4-Methoxy phenyl & 3.410 & 3.470 \\
\hline TSM32 & $-\mathrm{CH}_{3}$ & - 3,4-Dimethoxy phenyl & 3.330 & 3.539 \\
\hline TSM33 & $-\mathrm{CH}_{3}$ & - 3-Methyl phenyl & 3.800 & 3.677 \\
\hline
\end{tabular}

${ }^{*}$ Test set molecules and remaining are training set molecules, ${ }^{\#}$ Actual activity, ${ }^{* *}$ Predicated activity. 
Laxman Kawale et al.

Table 3: The dataset of seven test set molecules were sorted randomly from 33 reference compounds in the process of model refinement for CoMFA.

\begin{tabular}{llllllll}
\hline Sr. No. & $p E D_{25}{ }^{*}$ & $p E D_{25}{ }^{* *}$ & Residual pAlogit & SD $^{* * *}$ & ${\text { pAlogit }- \text { MATSM }^{@}}_{\text {SD }^{* * *}}$ & Predicated $^{2}$ \\
\hline TSM 9 $^{*}$ & 1.04 & 1.21 & 0.17 & 0.0289 & -1.10 & 1.210 \\
TSM 14 $^{*}$ & 1.93 & 1.37 & -0.56 & 0.3136 & -0.21 & 0.044 \\
TSM 27 $^{*}$ & 3.42 & 3.44 & 0.02 & 0.0004 & 1.28 & 1.638 \\
TSM 29 & 2.99 & 2.96 & -0.03 & 0.0009 & 0.85 & 0.722 & 0.052 \\
TSM 30 $^{*}$ & 2.37 & 2.31 & -0.06 & 0.0036 & 0.23 & 0.384 \\
TSM 4 $^{*}$ & 1.52 & 0.95 & -0.57 & 0.3249 & -0.62 & 1.690 \\
TSM 5 $^{*}$ & 0.84 & 1.30 & 0.46 & 0.2116 & -1.30 & \\
\hline
\end{tabular}

${ }^{*}$ Test set molecules, ${ }^{\#}$ Actual activity, ${ }^{* *}$ Predicated activity, ${ }^{* * *}$ Squared deviations, ${ }^{\circledR}$ Mean activity of training set molecules

Table 4: Statistical data of CoMFA analysis

\begin{tabular}{llll}
\hline Statistical parameters & CoMFA Analysis & Statistical parameters & CoMFA Analysis \\
\hline Number of reference compounds & 33 & Standard error of estimate & 0.127 \\
Molecule use for alignment & 33 & Non cross validated r squared & 0.986 \\
Training set molecules & 26 & F Value & 317.319 \\
Test set molecules & 7 & Probability of r squared & 0 \\
Number of outlier molecules & 0 & R squared predication & 0.741 \\
Cross validated r squared & 0.894 & Steric contribution & $73.00 \%$ \\
Optimum number of component & 5 & Electrostatic contribution & $27.00 \%$ \\
Column filtering use & 2 & & \\
\hline
\end{tabular}

Molecular alignment, i.e., molecular conformation and orientation, is one of the sensitive inputs for CoMFA. Protein-bound ligand confirmation of a template molecule 33 in the co-crystal structure of PPAR- $\gamma$ receptor (PDBID: 2PRG) was considered a bioactive conformation. A common substructure-based alignment method was used to align all reference compounds on a template reference molecule 33 (Fig. 1b). All the molecular modeling calculations were performed with the help of SYBYL 7.1 molecular modeling package installed on a Silicon Graphics Fuel Workstation running IRIX 6.5.

For aligned molecules, the steric and electrostatic CoMFA fields at each lattice intersection of a regularly spaced grid box of $2.0 \AA$ were calculated using the default probe, a sp3 carbon atom with a charge +1 and a Van der Waals radius of $1.52 \AA$. The Lennard-Jones potentials and Coulombic terms were calculated using the Tripos force field. The steric and electrostatic energies were truncated at $30 \mathrm{kcal} / \mathrm{mol}$. The minimum column filtering was set up to $2.0 \mathrm{kcal} / \mathrm{mol}$ to get a superior signal to noise ratio by omitting those lattice points whose energy variation was below this threshold.

Regression analysis was performed by using a leaveone-out method, a cross-validation analysis. The result from a cross-validated analysis was expressed as $r^{2}$ cvvalue. The non-cross-validated ( $\left.r^{2} \mathrm{ncv}\right)$ conventional analysis was produced with an optimal number of components 5 (from 7 test set molecules and remaining 2-test molecules are column filtering use) equal to that yielding the highest $r^{2} \mathrm{cv}$, and the corresponding conventional correlation coefficient $r^{2}$ ncv, standard error of estimate (SEE) and $F$ ratio were also calculated and reported in Table 4.

The predictive abilities were determined from a test set of 7 molecules. The $\mathrm{ED}_{25}$ values for test molecules were predicted by using the developed CoMFA model. Based on test set molecules, the predictive correlation coefficient ( $r^{2}$ pred) was calculated using following equation,

Where,

$$
\text { Predicated } r^{2}=(S D-P R E S S) / S D
$$

$\mathrm{SD}$ is the sum of squared deviations between the inhibitory activities of the test set molecules and mean activities of the training molecules,

PRESS is the sum of squared deviations between predicted and actual activity values for each molecule in the test set molecules.

\section{D-QSAR Analysis}

The resulting 3D-QSAR model analysis shows that the best CoMFA model was obtained by combining a steric and electrostatic field, which yields a r $2 \mathrm{cv}$ of 0.894 with five optimum numbers of components a $r^{2} \mathrm{ncv}$ of 0.986 , an estimated $F$ value of 317.319, and a low SEE of 0.127 . In this model, the steric and electrostatic contributions were found to be 73.00 and $27.00 \%$, respectively. The statistical parameters obtained from the CoMFA analysis are listed in Table 4 . The scattered plots of experimental $\mathrm{pED}_{25}$ against the CoMFA predicted $\mathrm{pED}_{25}$ of training and test set molecules were retorted in Figure 2. 
Hypoglycemic Activity of Newly Synthesis Sulphonyl Thiazolidine-2,4-diones.

\section{$3 D$ contour maps}

The final non-cross-validated PLS analysis and model of CoMFA has been used to generate the 3D contour maps. The contour maps illustrate lattice points and variation in the molecular field values at lattice points. It is strongly connected with variation in the receptor binding affinity. Molecular field analysis helped find out favorable and unfavorable interaction energies of aligned molecules with the aid of a probe atom, surrounding the molecules. These 3D contour maps (Figs. 3a and 3b) provide hints for the modification required to design new TZD's. Figure 3a showed green and yellow colors indicate that, $80 \%$ favorable and $20 \%$ unfavorable steric interactions, respectively. Figure $3 \mathrm{~b}$ showed blue and red colors, indicating $80 \%$ favorable and $20 \%$ unfavorable electrostatic interaction with TZD's.

\section{Feature selection procedure}

The best feature of 3D-QSAR was used to calculate predicted activities of all synthesized compounds and standard drugs such as rosiglitazone and pioglitazone. The result of these CoMFA models were subjected to external validation to get significant utility by predicting the activity of all synthesized compounds and standard drugs. All synthesized compounds and standard drugs were again constructed, minimized and aligned with template reference molecule 33 in an above similar manner. The predicted activities of all synthesized compounds and standard drugs are in good agreement with observed predicted activities within an acceptable error range and verified by the CoMFA $r^{2}$ pred value of 0.741 , mentioned in Table 4.

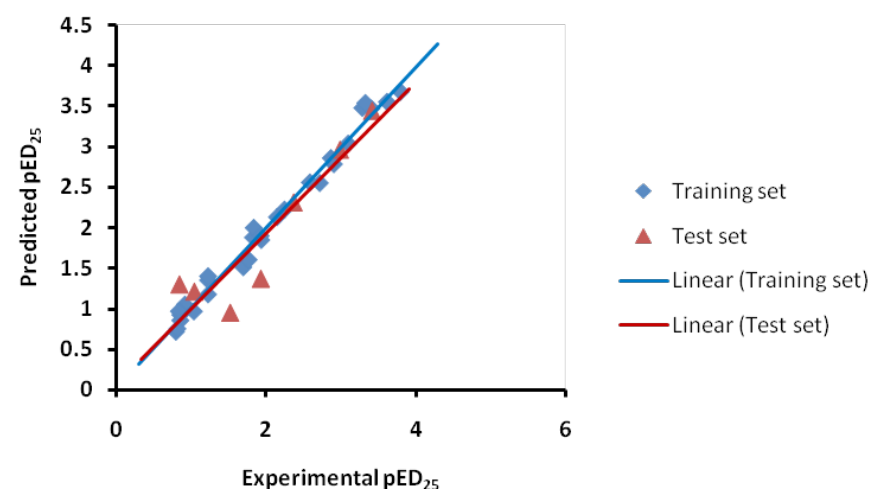

Fig. 2: The scattered plots

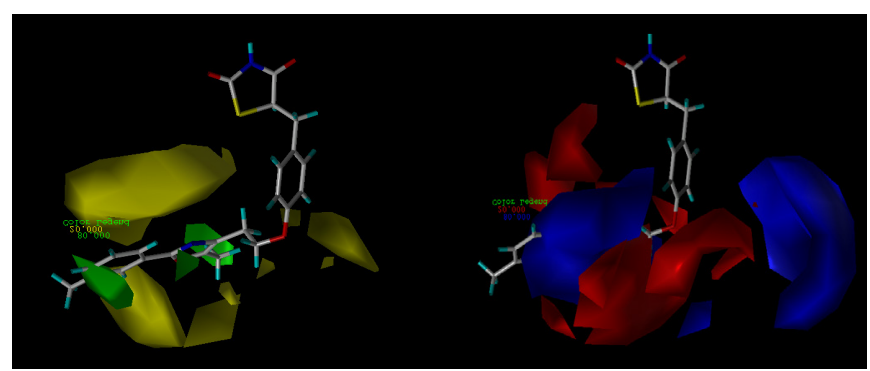

Fig. 3: 3D Contour map of a) steric field and b) electrostatic field distribution

\section{RESULTS AND DisCUSSION}

Synthesis of 5-[4-(substituted)sulphonylbenzylidene] thiazolidine-2,4-diones (4a-4h) were achieved with the help of different reactions such as Knoevenagel's condensation, chlorosulphonation and nucleophile displacement reactions (Scheme 1). These synthesized compounds were shown -NH- stretching frequency bands of thiazolidine-2,4-dione and sulphonamide in the IR regions between 3400 to $3150 \mathrm{~cm}^{-1}$, respectively. These final compounds also showed IR stretching frequency of 2 and 4 positions of carbonyl functional groups of thiazolidine-2,4-dione ring nearly at $1750 \mathrm{~cm}^{-1}$ and $1650 \mathrm{~cm}^{-1}$. Asymmetric and symmetric stretching IR frequency of sulphonamide containing $-\mathrm{SO}_{2}$-group of these compounds have shown IR stretching frequency bands at $1300 \mathrm{~cm}^{-1}$ and $1100 \mathrm{~cm}^{-1}$, respectively.

The ${ }^{1}$ HNMR spectra of synthesized compounds (4a-4h) showed a singlet signal of benzylidene proton in the range of 7.2 to $7.70 \delta \mathrm{ppm}$. These compounds also showed aromatic protons doublet-doublet signals in the range of 7.0 to $7.80 \delta \mathrm{ppm}$. The ${ }^{13} \mathrm{CNMR}$ spectra of some compounds ( $4 \mathrm{a}$ and $4 \mathrm{f}$ ) showed carbon of unsaturation $(-\mathrm{C}=\mathrm{CH}-$ ) signal in the range of $145-148 \delta \mathrm{ppm}$. The 2 and 4 positions of carbon signals of thiazolidine-2,4-dione ring of these compounds were shown in the range of 166 to $185 \mathrm{\delta ppm}$. Finally, molecular weight of synthesized compounds were confirmed by using mass spectrometry.

After synthesis and spectral interpretation of final prepared compounds ( $4 \mathrm{a}-4 \mathrm{~h})$, compounds $4 \mathrm{a}-4 \mathrm{~h}$ have been evaluated on streptozotocin-induced diabetes mellitus. The activity results of these compounds, significantly $(\mathrm{p}<0.05)$ reduced the rise in blood glucose levels in comparison with PCG, reported in Table 5. This oral hypoglycemic activity result of all synthesized compounds indicates that compounds with sulphonyl linkage have shown significant oral hypoglycemic activity as compared with PCG; if suitable substituted aniline moieties were present at the tail region of the synthesized molecules.

The results are expressed as mean \pm SEM. The data is analyzed using one-way Analysis of Variance (ANOVA)

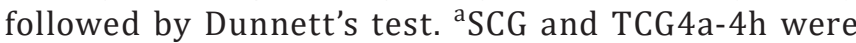
compared to PCG $(\mathrm{p}<0.05)$.

All synthesized compounds (4a-4h) were further studied to find out their hydrogen bonding interactions with 2PRG protein and it's docking score by using molecular docking software, reported in Table 6. The hydrogen bonding interactions results of all synthesized compounds (Figs. 4 to 7) were compared with standard drugs such as pioglitazone and rosiglitazone (Fig. 8), respectively. These comparable results suggested that, if His449, Tyr473, His323, Ser289 and Gln286 amino residues of PPAR- $\gamma$ protein interact with thiazolidine2,4-dione heterocyclic ring of TZD's were shown better binding affinity, docking score and producing significant oral hypoglycemic activity. 
Laxman Kawale et al.

Table 5: Oral hypoglycemic activity of synthesized compounds

\begin{tabular}{|c|c|c|c|}
\hline \multirow[b]{2}{*}{ Groups } & \multicolumn{3}{|c|}{ Blood glucose level $(\mathrm{mg} / \mathrm{dL})$} \\
\hline & $O h$ & $2 h$ & $5 h$ \\
\hline NCG & $131.00 \pm 08.718$ & $127.80 \pm 05.15$ & $127.20 \pm 02.88$ \\
\hline PCG & $336.20 \pm 20.21$ & $346.20 \pm 20.83$ & $343.00 \pm 21.48$ \\
\hline SCG & $330.00 \pm 17.77$ & $170.40 \pm 04.26^{\mathrm{a}}$ & $137.20 \pm 02.35^{\mathrm{a}}$ \\
\hline TCG4a & $314.20 \pm 21.48$ & $264.00 \pm 15.03^{d}$ & $220.80 \pm 14.7^{d}$ \\
\hline TCG4b & $307.00 \pm 13.56$ & $264.00 \pm 15.92^{a}$ & $196.00 \pm 16.39^{\mathrm{a}}$ \\
\hline TCG4c & $318.00 \pm 13.93$ & $248.00 \pm 11.14^{\mathrm{a}}$ & $209.00 \pm 13.82^{\mathrm{a}}$ \\
\hline TCG4d & $323.00 \pm 19.89$ & $250.00 \pm 7.42^{\mathrm{a}}$ & $196.40 \pm 23.82^{\mathrm{a}}$ \\
\hline TCG4e & $313.00 \pm 19.89$ & $214.00 \pm 7.42^{a}$ & $190.40 \pm 23.82^{\mathrm{a}}$ \\
\hline TCG4f & $335.80 \pm 17.22$ & $278.60 \pm 07.03^{a}$ & $196.80 \pm 04.21^{\mathrm{a}}$ \\
\hline TCG4g & $318.00 \pm 22.00$ & $273.00 \pm 22.34^{a}$ & $222.60 \pm 23.05^{a}$ \\
\hline TCG4h & $303.40 \pm 18.83$ & $262.80 \pm 17.89$ & $206.40 \pm 17.36$ \\
\hline
\end{tabular}

Table 6: Hydrogen bonding interaction and docking score of all synthesized compounds

\begin{tabular}{llll}
\hline Comp. & Interaction with 2PRG protein & Docking Score & pED $_{25}{ }^{*}$ \\
\hline $4 \mathrm{a}$ & Ser289 Tyr473 and Gln286 & -8.085 & 1.151 \\
$4 \mathrm{~b}$ & Ser289 Tyr473 and Gln286 & -8.497 & 1.240 \\
$4 \mathrm{c}$ & Ser289 Tyr473 and Gln286 & -8.190 & 1.286 \\
$4 \mathrm{~d}$ & Ser289 Tyr473 and Gln286 & -7.494 & 1.240 \\
$4 \mathrm{e}$ & Ser289 Tyr473 and Gln286 & -7.646 & 1.110 \\
$4 \mathrm{f}$ & Ser289 Tyr473 and Gln286 & -7.531 & 1.145 \\
$4 \mathrm{~g}$ & Ser289 Tyr473 and Gln286 & -8.555 & 1.140 \\
$4 \mathrm{~h}$ & Ser289 Tyr473 and Gln286 & -8.886 & 1.142 \\
Pioglitazone & His323, Ser289, Gln286, His449 and Tyr473 & -10.734 & 1.488 \\
Rosiglitazone & His323, Ser289, Gln286, His449 and Tyr473 & -11.353 & 1.421 \\
\hline
\end{tabular}

*Predicated activity $\mathrm{ED}_{25}$
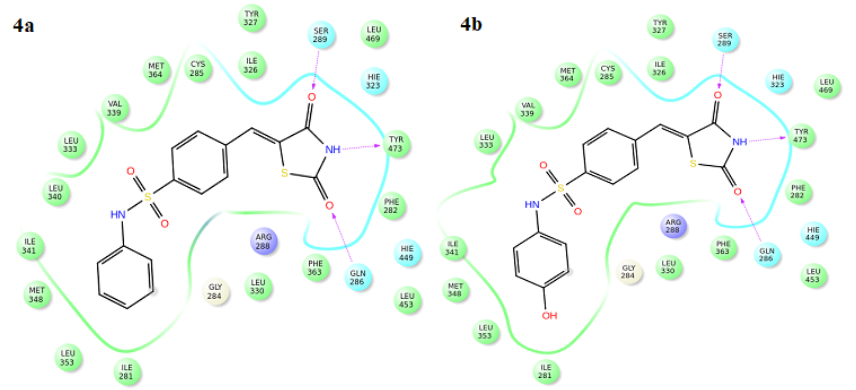

Fig. 4: Interaction of $4 \mathrm{a}$ and $4 \mathrm{~b}$ with different amino acids of $2 \mathrm{PRG}$ protein.

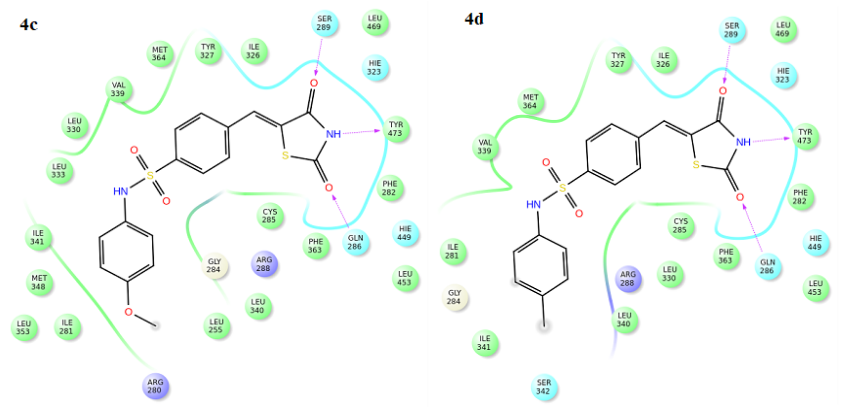

Fig. 5: Interaction of $4 \mathrm{c}$ and $4 \mathrm{~d}$ with different amino acids of $2 \mathrm{PRG}$ protein.

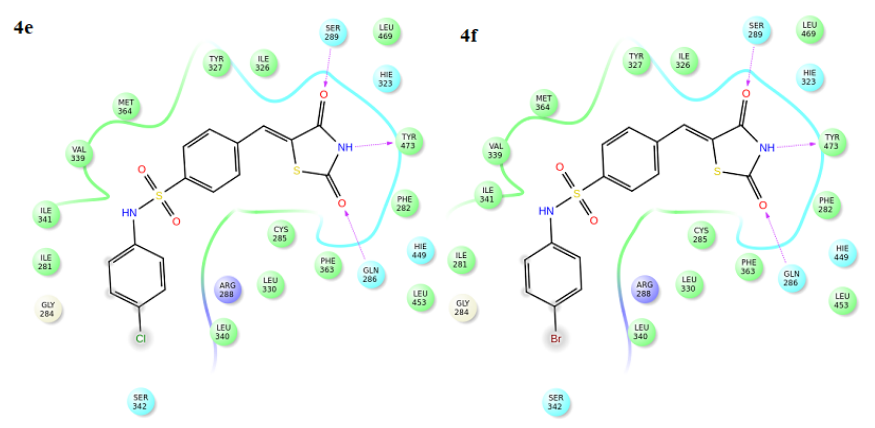

Fig. 6: Interaction of $4 \mathrm{e}$ and $4 \mathrm{f}$ with different amino acids of $2 \mathrm{PRG}$ protein.

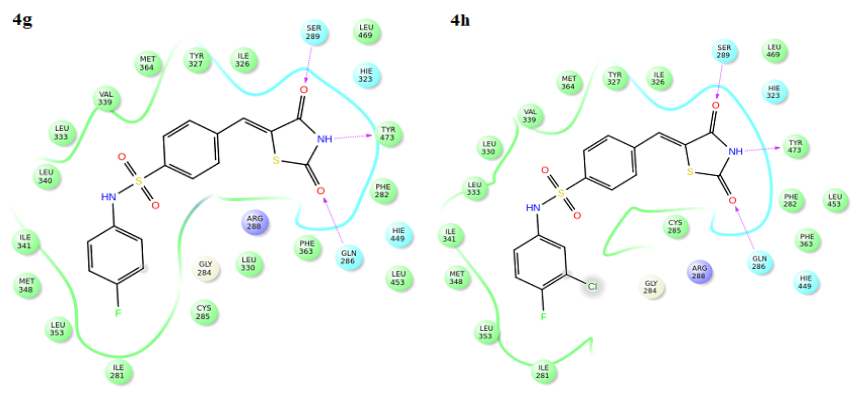

Fig. 7: Interaction of $4 \mathrm{~g}$ and $4 \mathrm{~h}$ with different amino acids of $2 \mathrm{PRG}$ protein. 
Hypoglycemic Activity of Newly Synthesis Sulphonyl Thiazolidine-2,4-diones.

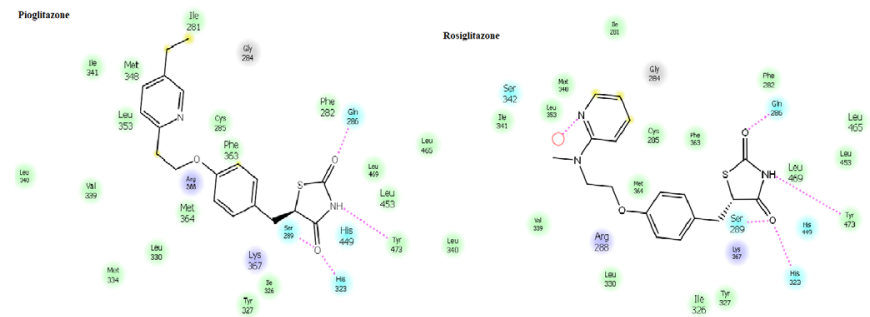

Fig. 8: Interaction of pioglitazone and rosiglitazone with different amino acids of 2PRG protein.

In addition, the predicted $\mathrm{pED}_{25}$ values of all synthesized compounds were reported in Table 6. The calculated predicted $\mathrm{pED}_{25}$ values of all synthesized compounds (4a-ah) were compared with standard drugs such as rosiglitazone and pioglitazone, respectively. All synthesized compounds were produced significant predicated $\mathrm{ED}_{25}$ values as compared with standard drugs. Finally, the overall results of molecular docking and 3D-QSAR studies concluded that, hydrogenbonding interactions with specific amino residues of PPAR- $\gamma$ with thiazolidine-2,4-dione heterocyclic ring were shown significant oral hypoglycemic activity and predicated $\mathrm{pED}_{25}$ values.

\section{CONCLUSION}

In the present work, 5-[4-(substituted)benzylidine] thiazolidine-2,4-diones were synthesized and evaluated for their oral hypoglycemic activity. The oral hypoglycemic activity result of all synthesized compounds indicated that, compounds with sulphonyl linkage have shown promising oral hypoglycemic activity; if suitable substituted amino moieties were present at the tail region of the synthesized compounds. Meanwhile, the docking score, hydrogen bonding interactions and predicted $\mathrm{pED}_{25}$ values of these synthesized compounds found out and compared with standard drugs, pioglitazone, and rosiglitazone.

The molecular docking study suggested that, if a specific amino residue of PPAR- $\gamma$ protein interact with thiazolidine-2,4-dione heterocyclic ring of TZD's, shown better binding affinity, docking score and significant oral hypoglycemic activity. In addition, the predicted $\mathrm{pED}_{25}$ values of all synthesized compounds were also calculated and compared with predicted $\mathrm{pED}_{25}$ values of standard drugs such as rosiglitazone and pioglitazone, respectively. All synthesized compounds were also produced significant predicated $\mathrm{ED}_{25}$ values as compared with standard drugs.

Finally, we concluded that suitable amino-substituted moieties and specific amino acid residues interaction of PAR- $\gamma$ protein with thiazolidine-2,4-dione heterocyclic ring of TZD's were required for better oral hypoglycemic activity.

\section{REFERENCES}

1. Al-Najjar BO, Wahab HA, Tengku Muhammad TS, Shu-Chien, AC, Ahmad Noruddin NA, Taha MO. Discovery of New Nanomolar Peroxisome Proliferator-Activated Receptor- $\gamma$ Activators Via
Elaborate Ligand-Based Modeling. Eur J Med Chem. 2011;46: 2513-2529.

2. Rikimaru K, Wakabayashi T, Abe H, Imoto H, Maekawa T, Ujikawa O, Murase K, Matsuo T, Matsumoto M, Nomura C, Tsuge H, Arimura N, Kawakami K, Sakamoto J, Funami M, Mol CD, Snell GP, Bragstad KA, Sang B-I, Dougan DR, Tanaka T, Katayama N, Horiguchi Y, Momose Y. A New Class of Non-Thiazolidinedione, NonCarboxylic-Acid-Based Highly Selective Peroxisome ProliferatorActivated Receptor (PPAR) $\gamma$ Agonists: Design and Synthesis of Benzylpyrazole Acylsulfonamides. Bioorg Med Chem. 2012; 20: 714-733.

3. Lohray BB, Bhushan V, Rao BP, Madhavan GR, Murali N, Rao KN, Reddy AK, Rajesh BM, Reddy PG, Chakrabarti R, Jajoo H, Vikramadithyan R, Rajagopalan R, Mamidi RNVS, Subramaniam S. Novel Euglycemic and Hypolipidemic Agents.1. J Med Chem. 1998; 41:1619-1630.

4. Xu Y, Rito CJ, Etgen GJ, Ardecky RJ, Bean JS, Bensch WR, Bosley JR, Broderick CL, Brooks DA, Dominianni SJ, Hahn PJ, Liu S, Mais DE, Montrose-Rafizadeh C, Ogilvie KM, Oldham BA, Peters M, Rungta DK, Shuker AJ, Stephenson GA, Tripp AE, Wilson SB, Winneroski LL, Zink R, Kauffman RF, McCarthy JR. Design and Synthesis of $\alpha$-Aryloxy- $\alpha$-Methylhydrocinnamic Acids: A Novel Class of Dual Peroxisome Proliferator-Activated Receptor $\alpha / \gamma$ Agonists. J Med Chem. 2004; 47:2422-2425.

5. Havale SH, Pal M. Medicinal Chemistry Approaches to the Inhibition of Dipeptidyl Peptidase-4 for the Treatment of Type 2 Diabetes. Bioorg Med Chem. 2009; 17:1783-1802.

6. Han HO, Kim SH, Kim K-H, Hur G-C, Yim HJ, Chung HK, Woo SH, Koo KD, Lee C-S, Koh JS, Kim GT. Design and Synthesis of Oxime Ethers of $\alpha$-Acyl- $\beta$-Phenylpropanoic Acids as PPAR Dual Agonists. Bioorg Med Chem Lett. 2007; 17: 937-941.

7. Kumar BRP, Soni M, Kumar SS, Singh K, Patil M, Nasir Baig RB, Adhikary L. Synthesis, Glucose Uptake Activity and StructureActivity Relationships of Some Novel Glitazones Incorporated with Glycine, Aromatic and Alicyclic Amine Moieties via Two Carbon Acyl Linker. Eur J Med Chem. 2011; 46:835-844.

8. Dumbare M, Kawale L, Nade V, Deshmukh R. Thiazolidine-2,4diones: An Update Review of Antidiabetic Agents. Int Res J Pharm. 2017; 8(12):12-29.

9. Elte J W F, Blicklé J F. Review Article Thiazolidinediones for the Treatment of Type 2 Diabetes. European Journal of Internal Medicine 2007; 18: 18-25.

10. Chiarelli F, Marzio D D. Peroxisome Proliferator-Activated Receptor- $\gamma$ Agonists and Diabetes: Current Evidence and Future Perspectives. Vascular health and risk management. 2008: 4(2): 297-304.

11. Dumbare M, Kawale L, Nade V, Deshmukh R. Review Article on Thiazolidine-2,4-Diones an Insulin Sensitizing Agents. Inter J of Pharmacotherapy. 2018; 8(2):39-53.

12. Roy A, Dumbare MR, Patil TD, Bhanwase AS, Deshmukh RD. Synthesis, Biological Evaluation and Molecular Modeling Studies of 5-[4-(Substituted)Benzylidene or Benzyl]Thiazolidine-2,4-dione with Oral Antihyperglycemic Activity. Int J Pharm Tech Res. 2013; 5(4):1882-1895.

13. Hulin B, Clark DA, Goldstein SW, McDermott RE, Dambek PJ, Kappeler WH, Lamphere CH, Lewis DM, Rizzi JP. Novel Thiazolidine2,4-diones as Potent Euglycemic Agents. J Med Chem. 1992. 35(10):1853-1864.

14. Nagarchi K, Ahmed S, Sabus A, Saheb SH. Effect of Streptozotocin on Glucose Levels in Albino Wister Rat. J Pharm Sci Res. 2015; 7: 67- 69.

15. Roy A, Dumbare MR, Harak SS, Najeeb MZ, Deshmukh RD. Synthesis, Molecular Docking Studies and Biological Evaluation of 5-[4-(Substituted)Benzylidene or Benzyl]Thiazolidine-2,4-dione with Their Oral Hypoglycemic Activity. Int Res J Pharm. 2013; 4:151-157.

16. Kulkarni SS, Gediya LK, Kulkarni V M. Three-Dimensional Quantitative Structure Activity Relationships (3-D-QSAR) of Antihyperglycemic Agents. Bioorg Med Chem. 1999; 7: 1475-1485.

HOW TO CITE THIS ARTICLE: Kawale L, Nade V, Deshmukh R, Dumbare M. Synthesis and Oral Hypoglycemic Activity of Some New Sulphonyl Linkage Thiazolidine-2, 4-diones. Int. J. Pharm. Sci. Drug Res. 2020;12(6):597-605. DOI: 10.25004/IJPSDR.2020.120603 